Quim. Nova, Vol. 34, No. 3, 516-519, 2011

\title{
DESENVOLVIMENTO E VALIDAÇÃO DE MÉTODO ANALÍTICO PARA DETERMINAÇÃO DO TEOR DE SINVASTATINA EM CÁPSULAS MAGISTRAIS
}

\author{
Hudson Caetano Polonini, Felipe Cerqueira dos Santos, Urias Pardócimo Vaz e Marcos Antônio Fernandes Brandão* \\ Departamento Farmacêutico, Faculdade de Farmácia, Universidade Federal de Juiz de Fora, Rua José Lourenço Kelmer, s/n, \\ 36036-330 Juiz de Fora - MG, Brasil
}

Nádia Rezende Barbosa Raposo

Departamento de Alimentos e Toxicologia, Faculdade de Farmácia, Universidade Federal de Juiz de Fora, Rua José Lourenço Kelmer, s/n, 36036-330 Juiz de Fora - MG, Brasil

Anderson de Oliveira Ferreira

Ortofarma Laboratório de Controle da Qualidade, BR 040, Empresarial Park Sul, 39, 36120-000 Matias Barbosa - MG, Brasil

Recebido em 23/4/10; aceito em 9/9/10; publicado na web em 8/12/10

\begin{abstract}
DEVELOPMENT AND VALIDATION OF ANALYTICAL METHOD FOR DETERMINATION OF SIMVASTATIN IN CAPSULES. Simvastatin is an oral anti-hyperlipidemic that has been widely used to reduce the cardiovascular disease risk. There isn't any pharmacopoeic method to assay this drug in capsules. It was proposed and validated a method for determining the content of simvastatin in capsules by UV/visible spectrophotometry $(\lambda=237 \mathrm{~nm})$, a more affordable method for the compounding pharmacy. The method was validated for linearity, specificity, range, accuracy, precision, performance, robustness, and limits of detection and quantification.
\end{abstract}

Keywords: simvastatin; spectrophotometry; validation studies.

\section{INTRODUÇÃO}

As doenças cardiovasculares (DC), dentre as quais figura a doença arterial coronariana (DAC), são um grande problema de saúde pública, uma vez que é a principal causa de morte no mundo. O Brasil acompanha este fenômeno internacional apresentando valores percentuais de mortalidade em torno de $25 \%$ para DC, o que representa cerca de 250.000 óbitos ao ano. ${ }^{1}$

Desde 1950, se conhece o risco que representam as taxas elevadas de colesterol plasmático para as doenças coronarianas, sendo seu controle plasmático ligado às proteínas de baixa densidade (LDLcol) fundamental para preveni-las. Desde então, a busca por agentes capazes de controlar as taxas de colesterol plasmático desperta o interesse das indústrias farmacêuticas. ${ }^{2}$

Nesse contexto, a sinvastatina insere-se como um agente hipocolesterolêmico amplamente utilizado. Trata-se de uma lactona inativa que é convertida em seu $\beta, \delta$-hidroxiácido correspondente (forma ativa), via metabolismo hepático pelo citocromo P450, após administração oral. Este fármaco (Figura 1) é um potente inibidor da enzima 3-hidroxi-3-metil-glutaril-coenzima A (HMGCoA) redutase, que catalisa a síntese de colesterol. ${ }^{1}$ É comercializada com o nome de Zocor ${ }^{\circledR}$ na forma de comprimidos revestidos nas concentrações de 10, 20, 40 e 80 mg e como Sinvastacor ${ }^{\circledR}$ na forma de comprimido revestido de $5 \mathrm{mg}^{3}$

Além das indústrias farmacêuticas, a sinvastatina também atraiu o interesse das farmácias magistrais, que atualmente manipulam extensivamente o fármaco na forma de cápsulas. Tais farmácias passaram, nos últimos anos, por um processo de expansão no número de medicamentos dispensados, o que pode acarretar riscos para o paciente, caso os requisitos de segurança, qualidade e eficácia não sejam comprovados. Desta forma, é essencial o controle de qualida-

\footnotetext{
*e-mail: marcosbrand@uol.com.br
}<smiles>CCC(C)(C)C(=O)OC1C[C@H](C)C=C2C=C[C@H](C)[C@H](CC[C@H]3C[C@H](O)CC(=O)O3)[C@H]21</smiles>

Figura 1. Estrutura química da sinvastatina (CAS 79902-63-9)

de do produto acabado nas farmácias de manipulação, pois um dos parâmetros que mais comumente apresentam desvios da qualidade é a quantidade de princípio ativo determinada no doseamento. ${ }^{4,5}$

Dentre as monografias de produtos acabados que constam nos compêndios oficiais reconhecidos pela Agência Nacional de Vigilância Sanitária pesquisados (Farmacopeias Brasileira, ${ }^{6}$ Norteamericana, ${ }^{7}$ Britânica, ${ }^{8}$ Europeia, ${ }^{9}$ Internacional - OMS, ${ }^{10}$ Japone$\mathrm{sa}^{11}{ }^{11}$ Mexicana ${ }^{12}$ e Portuguesa $\left.{ }^{13}\right)$, bem como em outras farmacopeias não oficiais no Brasil (Chinesa ${ }^{14}$ e Indiana $\left.{ }^{15}\right)$ não há descrição de método para a sinvastatina em cápsulas. A United States Pharmacopeia $32^{7}$ e a British Pharmacopoeia $2010^{8}$ possuem métodos para o doseamento em comprimidos, ambos por cromatografia líquida de alta eficiência (CLAE), uma técnica de elevado custo para as farmácias magistrais.

$\mathrm{Na}$ literatura consultada, são descritos métodos por cromatografia eletrocinética micelar, ${ }^{16}$ voltametria cíclica, ${ }^{17}$ CLAE, ${ }^{18-20}$ espectrofotometria direta ${ }^{21} \mathrm{e}$ espectrofotometria derivativa, ${ }^{22}$ porém todos destinados ao controle de comprimidos, e não cápsulas. Este fato inviabiliza a execução do controle de qualidade das cápsulas manipuladas por estes estabelecimentos. 
O objetivo deste trabalho foi validar um método espectrofotométrico rápido, seguro e de baixo custo para determinação do teor de sinvastatina em cápsulas.

\section{PARTE EXPERIMENTAL}

\section{Reagentes e padrão de referência}

Cápsulas contendo sinvastatina $(10 \mathrm{mg})$ foram gentilmente fornecidas por Singularis Farmácia de Manipulação Ltda. (São João Nepomuceno, MG). A sinvastatina, substância química de referência Deg (São Paulo, SP), foi padronizada de forma rastreável ao padrão primário da United States Pharmacopoeia (Rockville, MD). O solvente usado foi acetonitrila pró-análise (PA) Vetec (Rio de Janeiro, RJ).

A formulação das cápsulas foi: laurilsulfato de sódio $1,0 \%$; amidoglicolato de sódio 4,0\%; dióxido de silício coloidal 0,2\%; e lactose mono-hidratada malha 200: celulose microcristalina (3:1, p/p), qsp (quantidade suficiente para) $100,0 \%$.

Os espectros de absorção na região do ultravioleta foram obtidos em espectrofotômetro UV/visível de duplo feixe (Cary 50 Probe, Varian, Palo Alto, CA), com espectro de absorção de 190 a 1100 $\mathrm{nm}$ e cubeta de quartzo com caminho óptico de $1,0 \mathrm{~cm}$. O aparelho foi calibrado com acetonitrila PA e as absorvâncias foram lidas em comprimento de onda $(\lambda)$ de $237 \mathrm{~nm}$.

\section{Métodos}

\section{Doseamento de cápsulas de sinvastatina}

O conteúdo de 20 cápsulas magistrais de sinvastatina foi homogeneizado e uma quantidade de pó equivalente a $50 \mathrm{mg}$ de sinvastatina foi pesada, transferida para balão volumétrico de $100 \mathrm{~mL}$, solubilizada com acetonitrila e diluída com o mesmo solvente até o volume final do balão. A solução foi agitada mecanicamente por 10 min e filtrada em membrana de celulose com porosidade de $0,45 \mu \mathrm{m}$. Uma alíquota de $1 \mathrm{~mL}$ do filtrado foi então transferida para balão volumétrico de 50 $\mathrm{mL}$, o qual teve seu volume completado com acetonitrila (concentração final $\left.=10 \mu \mathrm{g} \mathrm{mL}^{-1}\right)$. A absorvância desta solução foi lida em 237 $\mathrm{nm}$, utilizando acetonitrila como branco. O teor de sinvastatina foi calculado pela comparação com a absorvância do padrão secundário preparado sob as mesmas condições descritas. Todos os experimentos foram conduzidos ao abrigo da luz.

\section{Parâmetros analíticos da validação}

Conforme a Resolução RE n. 899 , de $29 / 5 / 2003{ }^{23}$ que regulamenta a validação de métodos analíticos e bioanalíticos no Brasil, considera-se que este trabalho (determinação de teor) se encontra classificado na categoria de "Testes quantitativos para a determinação do princípio ativo em produtos farmacêuticos ou matérias-primas". Portanto, foram avaliados os seguintes parâmetros exigidos pela legislação para o método desenvolvido: linearidade e intervalo, especifidade e exatidão, precisão e robustez. Em caráter de complementação, foram determinados os limites de detecção (LD) e quantificação (LQ).

\section{Linearidade e intervalo}

$\mathrm{O}$ teste foi realizado a partir de três curvas analíticas, construídas a partir das concentrações de 80, 90, 100, 110 e 120\% (faixa de aceitação do produto), de forma a avaliar a relação linear entre a concentração do analito e as absorvâncias obtidas. Para tanto, os dados de cada nível de concentração foram estatisticamente avaliados através de análise de variância (ANOVA) e do valor do coeficiente de correlação da curva analítica. Dessa forma, a linearidade foi avaliada no intervalo de $80-120 \%$, ou seja, de $8-12 \mu \mathrm{g} \mathrm{mL}^{-1}$ de sinvastatina na solução final utilizada para leitura espectrofotométrica.

\section{$\underline{\text { Precisão }}$}

O teste de precisão teve o intuito de avaliar o grau de dispersão entre a série de medidas obtidas por um mesmo analista (repetibilidade) e entre dois analistas (precisão intermediária), para soluções na concentração de $100 \%$. A repetibilidade (precisão intraensaio) foi determinada pela análise de 6 replicatas de forma consecutiva e a precisão intermediária também foi realizada em 6 replicatas, mas em 2 dias, por analistas diferentes e em dois períodos do dia.

\section{Especificidade e exatidão}

Para determinação da especificidade e da exatidão do método foi realizado um teste de recuperação. A recuperação é a proporção da quantidade do analito que é passível de ser quantificada, sendo que a porcentagem de recuperação é obtida dividindo-se o teor medido do componente adicionado pelo valor efetivamente adicionado e multiplicado por $100 .^{24,25}$

Para essa determinação foram preparadas 5 soluções padrão de sinvastatina nas concentrações de $80,90,100,110$ e $120 \%$, todas com solução placebo (solução com excipiente). As leituras obtidas para estas soluções foram comparadas com uma solução padrão a $100 \%$ não contaminada (dita real), a fim de se avaliar a recuperação obtida.

\section{Robustez}

A robustez referente ao preparo da amostra foi avaliada e, para este intento, a legislação brasileira ${ }^{23}$ preconiza o estudo da estabilidade da solução analítica, o qual foi realizado com a solução contendo amostra de sinvastatina contaminada com placebo e da solução de padrão secundário, esta última sem contaminação de placebo. ${ }^{26}$ Este estudo foi realizado através da leitura das soluções imediatamente após o preparo e em intervalos específicos de tempo após o mesmo: $2,3,4$ e $5 \mathrm{~h}$.

\section{Limites de detecção e quantificação}

Os limites de detecção e quantificação do método proposto foram determinados a partir de três curvas de calibração do padrão e foram calculados a partir das Equações 1 e 2:

$$
\begin{gathered}
L D=S 3 / a \\
L Q=S 10 / a
\end{gathered}
$$

nas quais $\mathrm{S}$ é o desvio padrão do intercepto com o eixo y de três curvas de calibração e $a$ é o coeficiente angular da curva analítica.

\section{Análise estatística}

Foi realizada a análise descritiva dos dados, calculando-se a medida da tendência central (média) e medidas de dispersão (desvio padrão, desvio padrão relativo e coeficiente de variação) referentes aos parâmetros avaliados.

\section{RESULTADOS E DISCUSSÃO}

O intuito deste trabalho foi validar um método acessível ao setor magistral, ou seja, mais facilmente aplicado à rotina de controle da qualidade de produtos magistrais. Desta forma, foi desenvolvido um método espectrofotométrico para o doseamento de sinvastatina em cápsulas, pois a espectrofotometria no UV/visível é uma alternativa mais econômica e de mais fácil execução quando comparada à CLAE.

A varredura pelos comprimentos de onda de 200-300 nm de uma solução de cápsulas de sinvastatina em acetonitrila a $10 \mu \mathrm{g} \mathrm{mL} \mathrm{m}^{-1}$ forneceu o espectro de absorção visualizado na Figura 2. O espectro evidencia a não interferência dos excipientes na leitura espectrofotométrica. Pode-se visualizar o pico principal situado em $237 \mathrm{~nm}$, 
Tabela 1. Resultados do estudo da linearidade

\begin{tabular}{ccccccccc}
\hline $\begin{array}{c}\text { Concentração } \\
\left.(\mu \mathrm{g} \mathrm{mL})^{-1}\right) \\
80-120 \%\end{array}$ & $\begin{array}{c}\text { Abs média } \\
(\mathrm{n}=3)\end{array}$ & DPR $(\%)$ & CV $(\%)$ & Fonte de variação & GL & QM & Fo & $\mathrm{F}_{\text {crítico }}$ \\
\hline 8,08 & 0,431 & 0,002 & 0,48 & Regressão & 1 & 0,084 & 6120,16 \\
9,09 & 0,484 & 0,001 & 0,23 & Resíduo & 13 & 0,000 & 6,410 \\
10,10 & 0,542 & 0,002 & 0,42 & Linearidade & 3 & 0,000029 & 3,19 \\
11,11 & 0,593 & 0,001 & 0,09 & Erro Puro & 10 & 0,0000091 & 4,830 \\
12,12 & 0,642 & 0,006 & 0,91 & Total & 15 & 0,006 & \\
\hline
\end{tabular}

Abs = absorvância. DPR = Desvio-padrão relativo. CV = Coeficiente de variação. GL = Graus de liberdade. QM = Quadrado médio.

o qual serviu de base para o cálculo do teor pela comparação da absorvância entre padrão e amostra nesse comprimento de onda. O teor médio encontrado foi de $99,87 \%$.

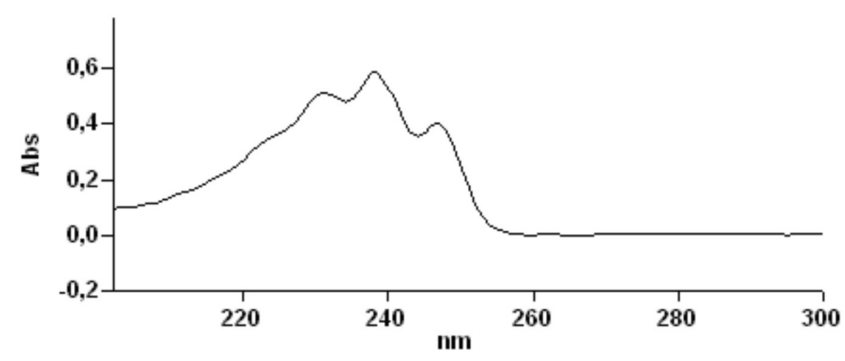

Figura 2. Espectro de absorção de cápsulas de sinvastatina no UV

A validação deste método possibilita que se afirme que o mesmo é uma ferramenta eficiente para o controle de qualidade de cápsulas de sinvastatina, de modo seguro e confiável analiticamente. Os parâmetros avaliados foram linearidade, limites de detecção e quantificação, precisão, especificidade, exatidão e robustez.

A RE 899/2003 ${ }^{23}$ preconiza que se avalie a relação linear entre os dados gerados durante o processo de validação, considerando-se que um método linear é aquele que gera resultados diretamente proporcionais à concentração do analito, dentro do intervalo especificado. ${ }^{27}$ Deve-se, então, submeter os resultados a testes estatísticos para determinação do coeficiente de correlação (r), intersecção com eixo y, coeficiente angular, soma residual dos quadrados mínimos da regressão linear e desvio padrão relativo.

Pelo método dos mínimos quadrados, obteve-se a equação da reta $y=0,0526 x+0,0074$. A análise da regressão linear demonstrou um coeficiente de determinação $\left(\mathrm{R}^{2}\right)$ muito próximo da unidade $(0,9991)$, o que sugere a linearidade do método.

Através da análise de variância, ${ }^{28,29}$ pode-se testar a linearidade do método e a significância estatística da curva ajustada. Utilizando a razão entre a média quadrática (falta de ajuste) e a média quadrática (erro puro) foi possível verificar se houve falta de ajuste (Tabela 1).

Através do teste $\mathrm{F}$, analisou-se a validade da regressão e o modelo linear da curva analítica. Assim, foram comparados valores de F tabelados e calculados. Para avaliar o comportamento linear da curva, foi comparado o $\mathrm{F}_{0}$ obtido para a linearidade com o $\mathrm{F}_{\text {crítico }}$ ou $\mathrm{F}_{\text {tabelado, }}$, de forma que para comprovar a linearidade se deve encontrar um valor de $\mathrm{F}_{0}$ menor que o $\mathrm{F}_{\text {crítico. }}$. Esta relação apresentou um $\mathrm{F}_{\text {calculado }}\left(\mathrm{F}_{0}\right)$ igual a 3,19, abaixo do valor crítico tabelado 4,83. Pode-se afirmar, portanto, com $95 \%$ de confiança, que o modelo é linear e está bem ajustado na faixa de concentração estudada.

Para a validade da regressão, também foram comparados valores de $\mathrm{F}_{0}$ e $\mathrm{F}_{\text {crítico }}$, de modo que para o coeficiente angular da curva ser diferente de zero se deve ter valores de $\mathrm{F}_{0}$ maiores que os de $\mathrm{F}_{\text {crítico }}$. $\mathrm{O}$ valor encontrado para $\mathrm{F}_{0}$ foi 6120,16 , muito maior do que o $\mathrm{F}_{\text {crítico }} 6,410$. Assim, concluiu-se que a inclinação da reta não é nula.

A partir da curva analítica foram estimados o limite de detecção, a menor concentração detectável de analito pelo método e o limite de quantificação, a menor concentração quantificável do mesmo.,26,27 Obtiveram-se os seguintes valores: $\mathrm{LD}=0,5879 \mu \mathrm{g} \mathrm{mL}^{-1}$ e $\mathrm{LQ}=$ $1,9597 \mu \mathrm{g} \mathrm{mL}^{-1}$, que demonstram a sensibilidade do método, comprovando que o mesmo é adequado aos objetivos propostos.

Outro parâmetro analítico avaliado, a precisão, mede o grau de variação dos resultados apresentados pelo método proposto. Segundo a RE 899/2003,,$^{23}$ os resultados obtidos para as medições da precisão não podem apresentar variações, que são expressas em coeficiente de variação, superiores a 5\%. Conforme pode ser visto nas Tabelas 2 e 3 , todos os resultados encontrados se mantiveram dentro do critério de aceitação, sendo o método preciso para ambos os tipos de precisão avaliados. Logo, pode-se afirmar que o método se apresenta preciso intracorrida e intercorrida.

Tabela 2. Resultados do estudo da precisão

\begin{tabular}{lccccccccc}
\hline & \multicolumn{4}{c}{ Dia 1} & \multicolumn{4}{c}{ Dia 2 } \\
\hline Período & \multicolumn{2}{c}{ Manhã } & \multicolumn{2}{c}{ Tarde } & \multicolumn{2}{c}{ Manhã } & \multicolumn{2}{c}{ Tarde } \\
Analista & 1 & 2 & 1 & 2 & 1 & 2 & 1 & 2 \\
Abs média & 0,564 & 0,595 & 0,595 & 0,602 & 0,564 & 0,595 & 0,595 & 0,602 \\
& & & & & & & & \\
DP & 0,005 & 0,002 & 0,004 & 0,003 & 0,005 & 0,002 & 0,004 & 0,003 \\
CV $(\%)$ & 0,8 & 0,2 & 0,5 & 0,4 & 0,8 & 0,2 & 0,5 & 0,4 \\
\hline
\end{tabular}

Abs $=$ absorvância. $n=6 . \mathrm{DP}=$ Desvio-padrão. $\mathrm{CV}=$ coeficiente de variação.

Tabela 3. Resultados do estudo aplicado da precisão

\begin{tabular}{lccccccccc}
\hline & \multicolumn{1}{c}{ Intradia } & \multicolumn{3}{c}{ Interdia } \\
\hline & \multicolumn{1}{c}{ Dia 1 } & Dia 1 & Dia 2 & Dia 2 & \multicolumn{2}{c}{ Dia 1 x Dia 2} \\
Analista & 1 & 2 & \multicolumn{1}{c}{1 x 2} & 1 & 2 & 1 & 2 \\
Absorvância & 0,577 & 0,561 & 0,569 & 0,589 & 0,579 & 0,598 & 0,578 & 0,580 \\
média (n=6) & & & & & & & & \\
DPR (\%) & 0,015 & 0,025 & 0,022 & 0,015 & 0,016 & 0,004 & 0,015 & 0,026 \\
CV (\%) & 2,58 & 4,46 & 3,80 & 2,59 & 2,85 & 0,66 & 2,67 & 4,46 \\
\hline
\end{tabular}

$\mathrm{DPR}=$ Desvio-padrão relativo. $\mathrm{CV}=$ coeficiente de variação.

O critério de aceitação para a recuperação obtida para que o método seja considerado exato e específico é de $\pm 2,0 \%,{ }^{30}$ ou seja, entre 98,0 e 102,0\%. Como a porcentagem de recuperação média foi de $99,88 \%$ (Tabela 4), considera-se o método como sendo específico.

De acordo com as normas do ICH (International Conference on Harmonization,${ }^{26}$ a exatidão pode ser inferida desde que a precisão, linearidade e especificidade sejam estabelecidas. Sendo assim, pode-se afirmar que o método proposto é exato, visto que estes três 
Tabela 4. Resultados do teste de recuperação

\begin{tabular}{ccc}
\hline $\begin{array}{c}\text { Concentração teórica } \\
(\%)\end{array}$ & $\begin{array}{c}\text { Concentração experimental } \\
\left(\mu \mathrm{gL} \mathrm{m}^{-1}\right)\end{array}$ & $\begin{array}{c}\text { Recuperação média } \\
(\%)\end{array}$ \\
\hline 80 & $8,09 \pm 0,05$ & $100,54 \pm 0,65$ \\
90 & $9,12 \pm 0,09$ & $100,25 \pm 0,34$ \\
100 & $10,12 \pm 0,07$ & $99,44 \pm 0,10$ \\
110 & $11,13 \pm 0,08$ & $99,62 \pm 0,33$ \\
120 & $12,14 \pm 0,04$ & $99,58 \pm 0,37$ \\
Média & - & 99,88 \\
\hline
\end{tabular}

Resultados expressos como média \pm desvio padrão $(n=3)$

Tabela 5. Resultados do estudo da estabilidade da solução analítica

\begin{tabular}{cccccc}
\hline Replicata & \multicolumn{5}{c}{ Absorvância } \\
& $0 \mathrm{~h}$ & $2 \mathrm{~h}$ & $3 \mathrm{~h}$ & $4 \mathrm{~h}$ & $5 \mathrm{~h}$ \\
\hline 1 & 0,609 & 0,603 & 0,604 & 0,603 & 0,683 \\
2 & 0,610 & 0,606 & 0,607 & 0,605 & 0,674 \\
3 & 0,613 & 0,609 & 0,600 & 0,603 & 0,673 \\
4 & 0,612 & 0,607 & 0,604 & 0,604 & 0,692 \\
5 & 0,612 & 0,605 & 0,603 & 0,605 & 0,672 \\
6 & 0,612 & 0,605 & 0,607 & 0,602 & 0,704 \\
Média & 0,611 & 0,606 & 0,604 & 0,604 & 0,683 \\
DP & 0,002 & 0,002 & 0,003 & 0,001 & 0,013 \\
CV (\%) & 0,246 & 0,337 & 0,437 & 0,201 & 1,880 \\
\hline
\end{tabular}

$\mathrm{DP}=$ Desvio-padrão. CV = Coeficiente de variação.

parâmetros foram avaliados. Pela Tabela 4, vê-se que tanto a recuperação média para cada nível de concentração quanto para a média de todos os níveis se situaram de 98,0-102,0\%. Assim, entende-se que o método é capaz de medir exatamente a sinvastatina mesmo em presença de outros compostos.

Para o estudo da robustez, foi avaliada a estabilidade da solução analítica. Pelos resultados mostrados na Tabela 5, vê-se que as soluções se mantiveram estáveis por até 4 h após o preparo. Apesar de ter havido interferência nas leituras no intervalo de tempo $5 \mathrm{~h}$, pode-se depreender que o método é robusto, pois dentro de $4 \mathrm{~h}$ foi possível determinar quantitativamente o fármaco, de modo que os teores encontrados foram próximos aos obtidos na determinação da precisão.

\section{CONCLUSÕES}

Nas condições descritas, o método espectrofotométrico para determinação quantitativa de sinvastatina em cápsulas se mostra de acordo com os parâmetros de validação exigidos pela legislação brasileira vigente. O método é, portanto, adequado para análises de rotina de controle de qualidade de cápsulas magistrais contendo sinvastatina, visto ser simples, rápido, seguro e de baixo custo.

\section{REFERÊNCIAS}

1. Carvalho, G. Q.; Alfenas, R. C. G.; Rev. Nutr. 2008, 21, 577.

2. Brasil, Ministério da Saúde; Portaria SAS n. 1015, de 20/12/2002, http:// sna.saude.gov.br/legisla/legisla/informes/SAS_P1.015_02informes.doc, acessada em Março 2010.

3. http://www.anvisa.gov.br/medicamentos/referencia/lista.pdf, acessada em Fevereiro 2010.

4. Meneghini, L. Z.; Adams, A. I. H.; Rev. Bras. Farm. 2007, 88, 67.

5. Baracat, M. M.; Montanher, C. L. S.; Kubacki, A. C.; Martinez, R. M.; Zonta, G. A. N.; Duarte, J. C.; Nery, M. M. F.; Gianotto, E. A. S.; Georgetti, S. R.; Casagrande, R.; Lat. Am. J. Pharm. 2009, 28, 427.

6. Farmacopéia Brasileira, $4^{\mathrm{a}}$ ed., Atheneu: São Paulo, 2003.

7. The United States Pharmacopeia, $32^{\text {nd }}$ ed., United States Pharmacopoeial Convention: Rockville, 2009.

8. British Pharmacopoeia 2010, The Stationery Office: London, 2009.

9. European Pharmacopoeia, $6^{\text {th }}$ ed., Council of Europe: Strasbourg, 2008.

10. The International Pharmacopoeia, $3^{\text {rd }}$ ed., World Health Organization: Geneva, 1994.

11. The Japanese Pharmacopoeia, $15^{\text {th }}$ ed., http://jpdb.nihs.go.jp/jp15e/, acessada em Junho 2010.

12. Farmacopea de los Estados Unidos Mexicanos, $8^{\text {a }}$ ed., Secretaría de Salud: México, D.F., 2004.

13. Farmacopeia Portuguesa, $8^{\mathrm{a}}$ ed., Infarmed: Lisboa, 2005.

14. Pharmacopoeia of the People's Republic of China, Peoples's Medical Publishing House: Beijing, 2005.

15. Indian Pharmacopoeia, Controller of Publications: Delhi, 1996.

16. Srinivasu, M. K.; Raju, A. N.; Reddy, G. O.; J. Pharm. Biomed. Anal. 2002, 29, 715

17. Coruh, Ö.; Özkan, S. A.; Pharmazie 2006, 61, 285.

18. Gandhimathi, M.; Ravi, T. K.; Varghese, A.; Ninan, A.; Ind. Drugs 2003, 4, 707 .

19. Abu-Nameh, E. S. M.; Shawabkeh, R. A.; Ali, A.; J. Anal. Chem. 2006, 61,63 .

20. Nováková, L.; Šatinský,D.; Solich, P.; TrAC, Trends Anal. Chem. 2008, $27,352$.

21. Arayne, M. S.; Sultana, N.; Hussain, F.; Ali, S. A.; J. Anal. Chem. 2007, $62,536$.

22. Wang, L.; Asgharnejad, M.; J. Pharm. Biomed. Anal. 2000, 21, 1243.

23. Agência Nacional de Vigilância Sanitária - ANVISA; RE nº 899, de 29/5/2003: Guia para validação de métodos analíticos e bioanalíticos, Ministério da Saúde: Brasil, 2003.

24. Valente Soares, L. M.; Rev. Inst. Adolfo Lutz 2001, 60, 79.

25. Ribani M.; Bottoli, C. B. G.; Collins, C. H.; Jardim, C. S. F.; Melo, L. F. C.; Quim. Nova 2004, 27, 771.

26. ICH; International Conference on Harmonization of Technical Requeriments for Registration of Pharmaceuticals for Human Use, Q2B(R1): Guideline on Validation of Analytical Procedure-Methodology, 2005.

27. Shabir, G. A.; J. Chromatogr., A 2003, 987, 57.

28. Montgomery, D. C. Em Design and Analysis of Experiments; Montgomery, D. C., ed.; Wiley: New York, 2000, p. 177-185.

29. Barros Neto, B.; Scarminio, I. S.; Bruns, R. E.; Como fazer experimentos: pesquisa e desenvolvimento na ciência e na indústria, $4^{\mathrm{a}}$ Ed., Bookman: Porto Alegre, 2010.

30. Asociación Española de Farmacéuticos de la Industria (AEFI); Validación de Métodos Analíticos, 2001, p. 23-125. 